ter voranbringen, die Wertegemeinschaft und mit dem Europäischen Parlament die Demokratie stärken. Unter dem Eindruck der US-Finanzkrise wird die Einheit gefestigt werden zulasten des von Blankart gefürchteten natürlichen Zentralismus. „Durch das Nebeneinander kooperativer Herrschaften entsteht Systemwettbewerb, der friedlich oder kriegerisch sein kann.“ (S. 195) Hoffentlich meint er das nicht ernst.

Der deutsche Föderalismus ist das Ergebnis machtpolitischer äußerer Einwirkungen. 1866 konnte Preußen noch die mit Österreich verbündeten Mitgliedstaaten des Deutschen Bundes annektieren und zu Provinzen degradieren, wie es zu gleicher Zeit im Siegeszug von Sardinien-Piemont Ministerpräsident Conte di Cavour tat. Schon 1867 beim Norddeutschen Bund, noch mehr 1871 musste Otto von Bismarck im nun vereinten kleindeutschen Reich den Bundesstaaten erhebliche Rechte lassen. Die europäischen Mächte hätten einen deutschen Zentralstaat nicht zugelassen. Nach einer Unitarisierungsphase in der Weimarer Republik und dem NS-Zentralismus waren es wieder äußere Mächte, diesmal die westlichen Besatzer, die einen deutschen Föderalismus durchsetzten. Von vornherein war er auf eine Mischung der Gewalten angelegt. „Nicht absolute Trennung, sondern gegenseitige Kontrolle, Hemmung und Mäßigung der Gewalten ist dem Verfassungsaufbau des Grundgesetzes zu entnehmen." 1 Welche Probleme dies im europäischen Mehrebenensystem verursacht, schildert Baier. Wie sehr gegenseitige Abhängigkeit besteht, zeigen die Verhandlungen der zweiten Föderalismuskommission. An eine Trennung, gar eine absolute, wie sie Blankart vorschwebt, ist nicht nur nicht zu denken - es würde diese Staaten sprengen.

Erich Röper

1 BVerfGE 34, S. 52 ff., S. 59.

\title{
Verfassungsgericht und Politik: Modellschreinerei statt „politischer“ Analyse, Methodenkompetenz als Handicap
}

Hönnige, Christoph: Verfassungsgericht, Regierung und Opposition. Die vergleichende Analyse eines Spannungsdreiecks, VS Verlag für Sozialwissenschaften, Wiesbaden 2007, 280 Seiten, $€ 34,90$.

Wenn es zutreffen sollte, was Bertolt Brecht seinem „Galilei“ in den Mund legt, dass „das einzige Ziel der Wissenschaft darin besteht, die Mühseligkeit der menschlichen Existenz zu erleichtern" (14. Szene), dann hätte die hier zu rezensierende Potsdamer Dissertation auf grandiose Weise ihr Ziel verfehlt. Sie macht es nämlich weder den Juristen, die sich von Amts wegen mit dem Thema „Verfassungsgericht“ zu befassen haben, noch jenen Sozialwissenschaftlern, die wenigstens randständig an diesem Thema interessiert sein sollten, leicht, in ihrer wissenschaftlichen Existenz befestigt, gar in ihren Literatur-Interessen befriedigt zu werden, von einem zu erwartenden „politischen“ Mehrwert der Lektüre ganz zu schweigen. Im Gegenteil: Sie verlangt jedem Leser ein hohes Maß an asketischer Selbstdisziplin ab, sollte der sich überhaupt dazu durchringen, bis zur letzten Seite durchzuhalten. Am Ende wird er sich fragen, was er an Einsichten in die Sache, gar grundlegenden Erkenntnissen zum Verhältnis von Regierung, Opposition und Verfassungsgericht wirklich hinzugewon- 
nen hat, die er bis dato sich nicht hatte aneignen können. Außerdem mutet Christoph Hönnige allen ernsthaft am Thema Interessierten zahllose argumentative Umwege zu, und meist sind es sogar recht abwegige Seitenpfade, die eingeschlagen werden müssen, bevor man am Ziel der Analyse anlangt.

Gleichwohl soll es sich um eine „einfache“ (S. 29) Aufgabe handeln, der in einem Vergleich auf die Schliche zu kommen der Autor sich vorgenommen hat. Es klingt tatsächlich mehr als simpel, ebenso lapidar wie trivial: „Verfassungsrichter entscheiden auf Grund ihrer politischen Einstellungen. Deshalb haben Regierung und Opposition ein Interesse, Verfassungsrichter so auszuwählen, dass sie möglichst viele dieser Überzeugungen mit ihnen teilen. Die Mehrheitsverhältnisse im Gericht zu Gunsten oder zu Lasten der Opposition entscheiden dann über Erfolg oder Misserfolg einer Klage. Dementsprechend klagt die Opposition eher, wenn sie die Mehrheit der Richter ausgewählt hat und ihr keine besseren Alternativen in Form von ausgeprägten Mitwirkungsrechten im Gesetzgebungsprozess zur Verfügung stehen. Sie handelt also rational, strategisch kalkulierend und vermeidet unnötige Niederlagen. Deutliche Indizien dafür sind nicht zuletzt die Verfahrensabläufe bei der Verfassungsrichterwahl“ (S. 21 f.).

Damit wäre in aller Kürze auch schon das „Spannungsdreieck“ umrissen, dem die Analyse gilt. Zu diesem Zweck kann der Autor aus der bunten, jedoch politisch höchst unterschiedlichen Fülle von 17 west- und osteuropäischen Ländern auswählen, die (inzwischen) alle über ein Verfassungsgericht verfügen. Aber nicht der Aufdröselung dieses gebündelten Makartstraußes gilt sein analytisches Interesse, sondern - freilich aus weitgehend unerfindlichen Gründen - nur dem deutschen und dem französischen „Fall“, das heißt dem Bundesverfassungsgericht (BVerfG) und dem Conseil Constitutionnel (CC). Um die Unterschiede im Klageverhalten zwischen den beiden Gerichten zu erklären, werden für den Zeitraum 1974 bis 2002 die jeweiligen Klagequoten ermittelt. Obwohl sie „möglicherweise" nicht die einzige erklärungsrelevante Variable ist, liegt die Quote der beklagten Gesetze in Frankreich bei 10,4 Prozent, während sie in Deutschland lediglich 2,5 Prozent beträgt. Diese Unterschiede in den Klagequoten der beiden Länder werden im Wesentlichen auf die „ausgeprägteren [parlamentarischen] Mitwirkungsrechte [der Opposition in Deutschland] im Vergleich zu Frankreich“ zurückgeführt, wie dies hypothetisch auch schon hatte „vorhergesagt" werden können (S. 223). Um nun dieses unterschiedliche Klageverhalten der beiden Länder vor ihren jeweiligen Verfassungsgerichten erklären zu können, rückt ihnen der Autor nach dem Verfahren des so genannten Most Different Systems Design zu Leibe (Kapitel 4 und andere). „Bewusst werden zwei sehr unähnliche Fälle verglichen, um die Robustheit des postulierten Zusammenhanges zu testen. Das Most Different Systems Design hebt auf die Analyse der Mikroebene ab, was dem grundsätzlichen Ansatz der Arbeit, individuelles und kollektives Verhalten zu testen [gemeint ist das Verhalten jedes einzelnen Verfassungsrichters beziehungsweise des Gerichts qua Institution], entgegenkommt." (S. 139) So sollen verallgemeinerungsfähige Ergebnisse herausgebracht, das heißt versucht werden, „die externe Varianz - Drittvariablen, die den unterstellten Zusammenhang in irgendeiner Weise beeinflussen - bestmöglich zu kontrollieren, um zu gewährleisten, dass sein theoretisch vermuteter und empirisch nachgewiesener Zusammenhang tatsächlich als ein allgemeingültiger Mechanismus gelten kann..." (ebenda; [Hervorhebungen vom Rezensenten]). Ein „Zusammenhang“ soll also als „Mechanismus“ behandelt werden!? Tatsächlich ist dies das grundsätzliche Dilemma des Autors: Es geht ihm um Mechanismen beziehungsweise Verfahrensarten und nicht um politische Sachzusammenhänge, die zwar in juridisch 
stilisierten Sprachspielen verpackt, aber auch nur so und nicht anders „verarbeitet“ werden, um dann erst als konsensfähige Lösung zu gelten - Ziel aller Auseinandersetzungen vor einem Verfassungsgericht.

Nicht erst an dieser Stelle beginnen die sprachlichen und analytischen Verständnisschwierigkeiten, die sich selbst einer wohlwollenden Rezeption der Arbeit in den Weg stellen, sondern auch die Verständigungsschwierigkeiten zwischen den Disziplinen, die in die Untersuchung des Themas der "Natur der Sache" nach, das heißt notwendigerweise involviert sind. Zu konstatieren ist nämlich, dass der Autor auf exzessive Weise die Mathematisierung der Politikwissenschaft einschließlich der ihm (recht spärlich) zu Gebote stehenden und (traditionellerweise) weitgehend normativ sich verstehenden Jurisprudenz betreibt. Dass er zum Beispiel die (inzwischen historisch obsoleten) Staatsrechtslehren eines Carl Schmitt und Hans Kelsen, die doch den politischen Konflikt um Rolle und Funktion der Verfassungsgerichtsbarkeit in der Weimarer Republik exemplarisch widerspiegeln, immerhin einer Erwähnung für wert erachtet, ist allerdings kaum mehr als semantische Kosmetik, wenn nicht bloßes Alibi. Seine knappen Erörterungen zu ihnen bleiben jedenfalls im Hinblick auf die aktuelle Situation der Verfassungsgerichtsbarkeit weitgehend folgenlos. Stattdessen zwingt er die beteiligten Wissenschaftsdisziplinen - und ihre Vertreter - ins Prokrustesbett eines Methoden und analytische Verfahren fetischisierenden Perfektionismus.

Um dieses analytische Zwangsverhältnis, das die Lektüre seiner Arbeit durchgängig belastet, in aller Kürze zu illustrieren, sei eine typische Textpassage vorgestellt, nicht um die Darstellungsweise des Autors zu ironisieren, sondern um die Schwierigkeiten zu illustrieren, die seiner Lektüre, erst recht deren Verständnis im Wege stehen dürften. Welcher Jurist, welcher allgemein Interessierte, wenn er denn über die Verfassungsgerichtsbarkeit sich informieren möchte, belegt zuerst einmal Nachhilfekurse in beschreibender Statistik oder multivariaten Analysemethoden - zumal wenn er nicht sicher sein kann, dass ihn die Lektüre eines solchen Buches vor allem in sachlich-materieller Hinsicht, um deren Erhellung er ja bemüht war, schlauer macht, als er ursprünglich war.

So heißt es zum Beispiel in einem Zusammenhang, in dem das Modell oppositioneller Klagen und Erfolge dargestellt werden soll (S. 65 - 99), im Unterkapitel 3.1.2(4), in dem die „Präferenzmengen, Indifferenzkreise, Winsets und Distanzen“ zur Sprache kommen: „Akteure wählen von zwei Policy-Alternativen diejenige aus, die ihnen einen höheren Nutzen bringt, weil sie dichter an ihrem Idealpunkt liegt. Ob beispielsweise das Gericht ein neues Regierungsgesetz akzeptiert, hängt davon ab, ob es dichter an seinem Idealpunkt liegt als das bisherige Gesetz, der Status Quo. Alle Punkte, die sich auf der Nutzenfunktion um Akteur G durch den Status Quo befinden, stellen das Gericht genau so gut wie der Status Quo. Dieser Kreis wird auch Indifferenzkreis genannt. Alle Gesetzesvorschläge, die das Gericht gleich gut oder besser stellen als der Status Quo, bilden seine Präferenzmenge zum Status Quo. Diese wird formuliert als $\mathrm{P}_{\mathrm{G}}(\mathrm{SQ})$. Müssen in einem aus drei Richtern bestehenden Gericht alle drei Richter einstimmig entscheiden, besteht die Präferenzmenge $\mathrm{P}_{\mathrm{G}}(\mathrm{SQ})$ des Gerichtes aus der Überlappung der Präferenzmengen $\mathrm{P}_{\mathrm{J} 1}(\mathrm{SQ}) \cap \mathrm{P}_{\mathrm{J} 2}(\mathrm{SQ}) \cap \mathrm{P}_{\mathrm{J} 3}(\mathrm{SQ})$ der drei Richter. Damit bilden die überlappenden Präferenzmengen einen Kompromissraum, das sogenannte Winset, kurz notiert als $W\left(\mathrm{~J}_{1} \mathrm{~J}_{2} \mathrm{~J}_{3}\right)$. Der Kompromissraum zwischen Regierung und Gericht definiert sich als $\mathrm{W}(\mathrm{RG})=\mathrm{P}_{\mathrm{G}}(\mathrm{SQ}) \cap \mathrm{P}_{\mathrm{R}}(\mathrm{SQ})$. Gibt es keine Überschneidungsmenge zwischen zwei Akteuren, ist deren Winset leer: $\mathrm{W}(\mathrm{RG})=\varnothing$. Räumliche Distanzen zwischen zwei Akteuren, beispielsweise zwischen R und G, werden folgendermaßen ausgedrückt: | RG |.“ (S. 69) 
Während sich Hönnige immer wieder mit Problemen so genannter räumlicher Modelle abplagt, im Übrigen unter Zuhilfenahmen von 25 Abbildungen und 36 Tabellen, nur zu dem Zweck, die persönlichen Präferenzen von Verfassungsrichtern „als Positionen im politischen Raum“ abbilden zu können, bleibt dieser „politische Raum“ selbst weitgehend abstrakt, das heißt ohne jeden politischen Bedeutungsgehalt, also leer, weil er auf bloß mathematische Operationen reduziert erscheint. Damit aber hat sich das positivistische Verfahren, dessen sich der Verfasser durchgängig befleißigt, endgültig verselbständigt: rücksichtslos gegenüber dem Leser und ohne auf die in Frage stehende „Sache“ irgend Bezug zu nehmen, das heißt ausschließlich um Optimierung der methodischen Prämissen und deren messtechnische Nutzanwendung bemüht.

Wie lässt Brecht den Neuerer „Galilei“ gegen Ende seines Gelehrtendramas räsonieren? „Wenn Wissenschaftler (...) sich damit begnügen, Wissen um des Wissens willen aufzuhäufen, kann die Wissenschaft zum Krüppel werden." Dem ist im Falle der Hönnige-Dissertation nichts hinzuzufügen.

Otwin Massing

\section{Stärkung der pluralistischen und partizipativen Demokratie: Aufsatzsammlung mit Programm}

Eisfeld, Rainer: Streitbare Politikwissenschaft. Studien zu Demokratisierung, politischer Kultur und wissenschaftlicher Verantwortung. Mit einer Einführung von Michael Th. Greven, Nomos Verlagsgesellschaft, Baden-Baden 2006, 348 Seiten, € 49,-.

Anlässlich des vierzigjährigen Jubiläums des Protestjahres 1968 ist der Streit über dessen angemessene Deutung mit einer überraschenden Schärfe neu entbrannt. Nichts allerdings markiert die Deutungshegemonie der Generation der so genannten 68er in dieser Frage mehr als die Tatsache, dass die lautstärksten Protagonisten der heute scharf aufeinander prallenden Bewertungen damals gemeinsam zum kleinen Kreis der Protestaktivisten gehörten. Wie wenig sich bei einigen der damals Beteiligten Ton und Stil verändert haben, illustrierte zuletzt Götz Aly in seiner Abrechnungsschrift mit dem provokanten Titel „Unser

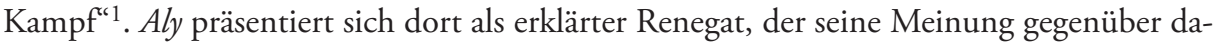
mals grundlegend geändert hat - aber er sieht sich schon wieder im Recht und sein Tonfall ist wieder von einer Einseitigkeit und Provokationslust geprägt, die bewusst darauf setzt, seine Leser wütend zu machen.

Vor dem Hintergrund einer solchen Debattenkultur bieten die in dem Buch, Streitbare Politikwissenschaft' zusammengestellten Aufsätze von Rainer Eisfeld eine Einladung zum Nachdenken und Abwägen. Eisfeld verficht ein Verständnis von ,kritischer Politikwissenschaft', das ohne gesellschaftstheoretische oder sozialphilosophische Anleihen auszukommen versucht (dessen konzeptionelle Vorteile listet Michael Th. Greven in seinem Vorwort auf, S. 7 - 16) und eher auf eine Schulung der politischen Urteilskraft abzielt.

Der Band enthält insgesamt 16 Beiträge des 1941 geborenen und seit 1974 in Osnabrück lehrenden Politikwissenschaftlers aus einer Zeitspanne von mehr als 35 Jahren,

1 Götz Aly, Unser Kampf: 1968 - ein irritierter Blick zurück, Frankfurt am Main 2008. 